\title{
Forming Mechanizm of Bhalekar-Gejji Chaotic Dynamical System
}

\author{
Sachin B. Bhalekar
}

Department of Mathematics, Shivaji University, Kolhapur, 416004, India

\begin{abstract}
Chaotic dynamical systems are used to model various natural phenomena. Bhalekar-Gejji chaotic dynamical system is a systemof three ordinary differential equations containing only two nonlinear terms. This system shows two-scroll butterfly-shaped attractor for certain values of para meters. In this article we show that the two-scroll attractor in this system is formed from two one-scroll attractors. We have used a control parameter in the third equation of the system to study the forming procedure of the attractor.
\end{abstract}

Keywords Chaos, Attractor, Limit Cycle, Synchronization

\section{Introduction}

Chaos is a phenomena observed in certain nonlinear dynamical systems. It is observed in a wide variety of systems such as Chua's circuit[1] in electronics, Belousov-Zhabotinsky reaction $[2,3]$ in chemistry, economics and finance[4-6], Ray leigh-Benard convection[7] in fluid dynamics, population dynamics[8], physiology[9, 10], pharmacodynamics [11] and meteorology [12].

E. N. Lorenz was the first to observe chaos in nonlinear system of differential equations. Lorenz system[12] represents convective motion of fluid which is cooled from above and warmed from below[13]. Few important examples of chaotic systems include Rossler system[14], Chen system[15], Liu system[16] and Lu system[17].

Chaotic trajectories are very sensitive to initial conditions i.e. the trajectories starting nearby could have comp letely different future. Though there is unpredictability, it is possible to make the behaviour of two (or many) nearby starting trajectories identical after some time period. This process is done by applying a suitable control and is termed as a synchronization. Examples of synchronization are abundant in nature. For the detailed discussion on this topic, readers are referred to[18-20]. Synchronization of chaotic systems have applications in secure communication[21]. Due to unpredictability, the crypto-systems based on chaotic synchronization are difficult to decode. The review on this topic is available in [22].

In this article we show that the two-scroll attractor in Bhalekar-Gejji system is formed from two one-scroll

* Corresponding author:

sachin.math@yahoo.co.in (Sachin B. Bhalekar)

Published online at http://journal.sapub.org/ajcam

Copyright (C 2012 Scientific \& Academic Publishing. All Rights Reserved attractors. We have used a control parameter in third equation of the proposed system to study the forming procedure of the attractor.

\section{Bhalekar-Gejji System}

A new chaotic system[23] proposed by Bhalekar and Daftardar-Gejji is given by the system of three ordinary differential equations.

$$
\begin{gathered}
\dot{x}=\omega x-y^{2}, \\
\dot{y}=\mu(z-y), \\
\dot{z}=\mathrm{a} \mathrm{y}-\mathrm{b} \mathrm{z}+\mathrm{x} \mathrm{y},
\end{gathered}
$$

where $\omega, \mu, \mathrm{a}, \mathrm{b}$ are constant parameters. System (2.1) shows a chaotic behaviour for $\omega=-2.667, \mu=10, a=27.3$, $\mathrm{b}=1$ as shown in Fig. 1 .

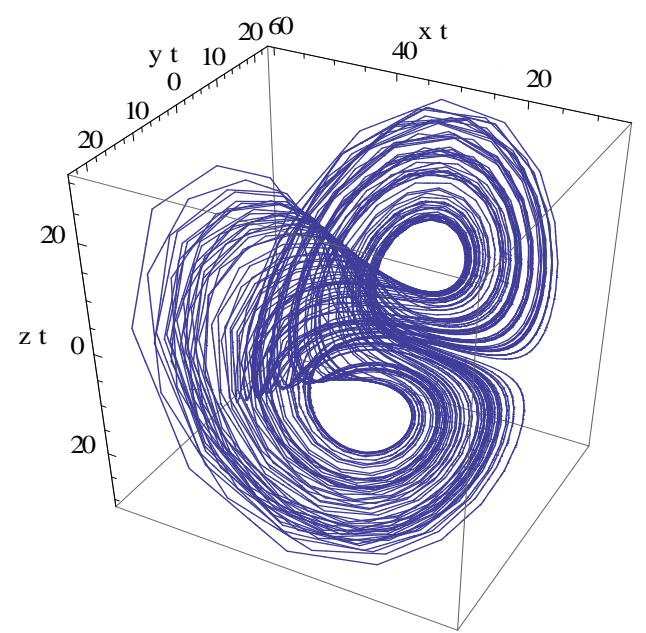

Figure 1. Chaotic phase portrait of (2.1)

Equilibrium points of the system (2.1) are given by the solutions of

$$
\omega x-y^{2}=0
$$




$$
\begin{gathered}
\mu(z-y)=0, \\
\mathrm{a} y-\mathrm{b} z+\mathrm{x} y=0,
\end{gathered}
$$

In Table 1, equilibrium points and corresponding eigenvalues of the Jacobian matrix

$$
J(x, y, z)=\left(\begin{array}{ccc}
\omega & 2 y & 0 \\
0 & -\mu & \mu \\
-y & a-x & -b
\end{array}\right)
$$

are listed for the parameter values $\omega=-2.667, \mu=10$,

\begin{tabular}{|c|c|c|}
\hline Equilibrium point & Eigenvalues & Nature \\
\hline $\mathrm{O}(0,0,0)$ & $-22.6245,11.6245,-2.667$ & $\begin{array}{l}\text { point } \\
\text { of index } 1 \\
\text { Saddle }\end{array}$ \\
\hline$E_{1}(26.3,-8.3751,-8.3751)$ & $-16.8614,1.5972 \pm 8.9804 i$ & $\begin{array}{c}\text { point } \\
\text { of index } 2\end{array}$ \\
\hline $\mathrm{E}_{2}(26.3,8.3751, .3751)$ & $-16.8614,1.5972 \pm 8.9804 i$ & $\begin{array}{c}\text { Saddle } \\
\text { point } \\
\text { of index } 2\end{array}$ \\
\hline
\end{tabular}
$\mathrm{a}=27.3, \mathrm{~b}=1$.

Table 1. Equilibrium points and corresponding eigenvalues

An equilibrium point $p$ of the system (2.1) is called a saddle point if the Jacobian matrix at $p$ has at least one eigenvalue with negative real part (stable) and one eigenvalue with non-negative real part (unstable). A saddle point is said to have index one (/two) if there is exactly one (/two) unstable eigenvalue/s. It is established in the literature [24-27] that, scrolls are generated only around the saddle points of index two. Saddle points of index one are responsible only for connecting scrolls.

\section{Forming Mechanism of Attractor}

In order to study the compound structure of the new attractor, we add a constant gain to the third equation.

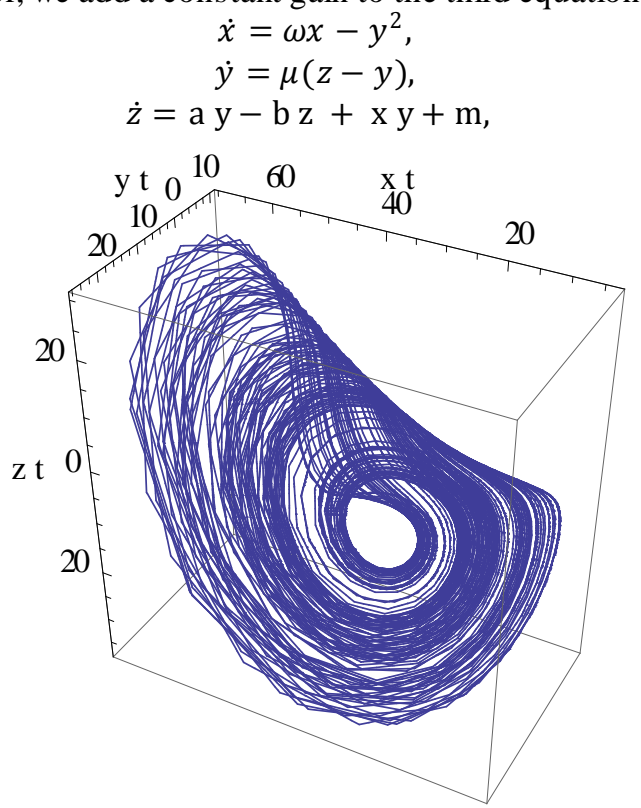

Figure 2(a). Left attract or $m=18.5$

We get one-scroll right-attractor for $m=18.5$ (cf. Fig. 2(a)) whereas $m=-18.5$ gives the mirror image of the right-attractor i.e. the left-attractor as shown in Fig. 2(b). Thus, the new attractor is a compound structure obtained by merging together two simple one-scroll attractors.

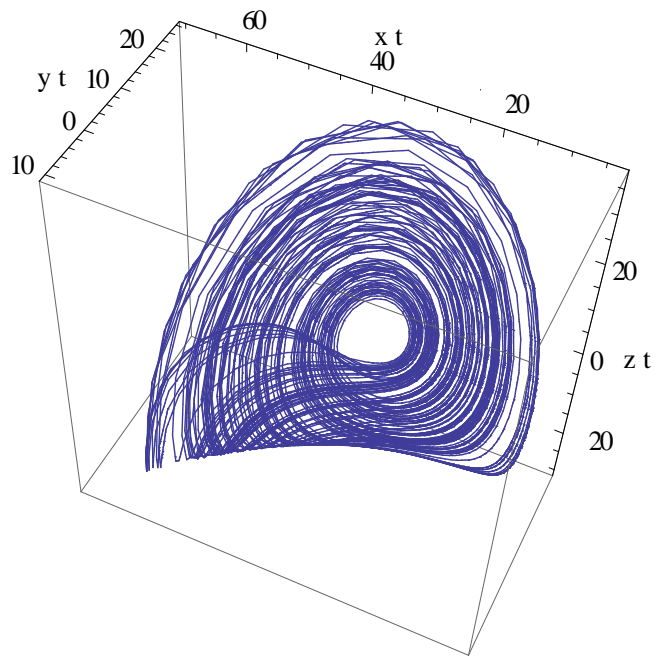

Figure 2(b). Right attractor $m=-18.5$

Now we study the behavior of the controlled system (3.1) for different values of parameter $m$.

- $|\mathrm{m}|<3.2$

The system is chaotic and shows double-scroll complete attractor.

$\bullet|\mathbf{m}|<4.8$

The system shows limit cycles for this range. In Fig. 3(a), the limit cycle is shown for $m=3.2$.

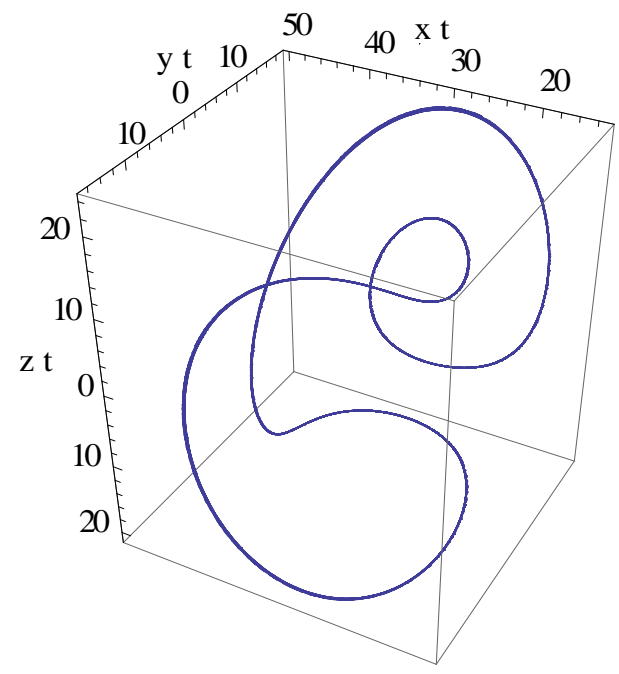

Figure 3(a). Limit cycle for $m=3.2$

- $|\mathbf{m}|<\mathbf{1 1}$

The system again shows complete attractor.

- 11.1 $\leq|\mathrm{m}|<\mathbf{1 1 . 4}$

The periodic window is observed in this range.

- 11.4 $\leq|\mathrm{m}|<18.5$

A partial attractor (cf. Fig. 3(b), m=14) is observed for these parameter values.

- $18.5 \leq|\mathrm{m}|<18.7$

Now, the system shows one-scroll (left or right) attractors. 
$\bullet 18.7 \leq|\mathrm{m}|<19.5$

Periodic limit cycles are observed in this range of parameter.

- $19.5 \leq|\mathrm{m}|<22.1$

One-scroll attractors are observed in this range.

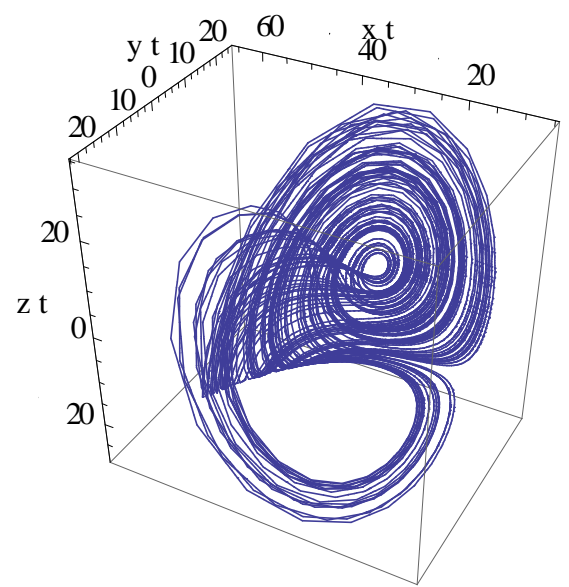

Figure 3(b). Partial attractor $m=14$

\section{Conclusions}

In this article, the forming mechanis m of Bhalekar-Gejji chaotic system is discussed. It is observed that the two-scroll attractor in the Bhalekar-Gejji system is formed from two one-scroll attractors. For this study, we have introduced a control parameter $\mathrm{m}$ in the third equation of the system. The complete double-scroll attractor observed for $|\mathrm{m}|<3.2$ is transformed to a partial attractor in the range $11.4 \leq|\mathrm{m}|<18.5$. Limit-cycles are also observed for certain values of parameter $\mathrm{m}$.

\section{REFERENCES}

[1] T. Matsumoto, A chaotic attractor from Chua's circuit, IEEE Trans. Circuits Syst., 31(12) (1984) 1055-1058.

[2] K. I. Agladze, V. I. Krinsky, A. M. Pertsov, Chaos in the non-stirred Belousov-Zhabotinsky reaction is induced by interaction of waves and stationary dissipative structures, Nature 308 (1984) 834-835.

[3] R. J. Field, L. Gyorgyi, Chaos in chemistry and biochemistry, world scientific, sin gapore, 1993.

[4] A. Serletic, Is there chaos in economic series?, Can. J. Econ., 29 (1996) S210-S212.

[5] W. C. Chen, Nonlinear dynamics and chaos in a fractional-order financial system, Chaos Solitons \& Fractals 36 (2008) 1305-1314.

[6] L. Fanti, P. Manfredi, Chaotic business cycles and fiscal policy: An IS-LM model with distributed tax collection lags, Chaos Solitons \& Fractals 32 (2007) 736-744.

[7] H. Xi, J. D. Gunton, Spatiotemporal Chaos In a Model of Rayleigh-Benard Convection, Phy. Rev. E, 52(4) (1995) 4963-4975.
[8] M.P. Hassell, H. N. Comins, R. M. May, Spatial structure and chaos in insect population dynamics, Nature, 353 (1991) 255-258.

[9] Basar, Erol (Ed), Chaos in brain function, Springer-Verlag, New York 1990.

[10] A. Eidukaitis, G. Varoneckas, D. emaitytee, Application of chaos theory in analyzing the cardiac rhythm in healthy subjects at different sleep stages, Human Physiology, 30(5) (2004) 551-555.

[11] A. Dokoumetzidis, A. Iliadis, P. Macheras, Nonlinear dynamics and chaos theory: concepts and applications relevant to pharmacody namics, Pharmaceutical Research, 18 (4) 2001 .

[12] E. N. Lorenz, Deterministic nonperiodic flow, J. Atmos. Sci., 20 (1963) 130.

[13] K. T. Alligood, T. D. Sauer, J. A. Yorke, Chaos: An Introduction to Dynamical Systems, Springer, New York, 2008.

[14] O.E. Rossler, An equation for continuous chaos, Phys. Lett. A 57 (1976) 397-398.

[15] G. Chen, T. Ueta, Yet another chaotic attractor, Int. J. Bifur. Chaos 9 (1999) 1465-1466.

[16] C. Liu, L. Liu, T. Liu, A novel three-dimensional autonomous chaos system, Chaos Solitons \& Fractals, 39(4) (2009) 1950-1958.

[17] J. H. Lu, G. R. Chen, A new chaotic attractor coined, Int. J. Bifurc. Chaos 12(3) (2002) 659-661.

[18] E. Mosekilde, Y. Maistrenko, D. Postnov, Chaotic synchronization: applications to living systems, World Scientific, Sin gapore, 2002.

[19] L. M. Pecora, T. L. Carroll, Synchronization in chaotic systems, Phys. Rev. Lett. 64(8) (1990) 821.

[20] L. M. Pecora, T. L. Carroll, Driving systems with chaotic signals, Phys. Rev. A 44 (1991) 2374.

[21] R. He, P. G. Vaidy a, Implementation of chaotic cryptography with chaotic synchronization, Phy. Rev. E 57(2) (1998) 1532-1535.

[22] S. Boccaletti, J. Kurths, G. Osipov, D.L. Valladares, C.S. Zhou, The synchronization of chaotic systems, Phys. Reports 366 (2002) 1-101.

[23] S. Bhalekar, V. Daftardar-Gejji, A new chaotic dynamical system and its synchronization, Proceedings of the international conference on mathematical sciences in honor of Prof. A. M. Mathai, 3-5 January 2011, Palai, Kerla- 686 574, India.

[24] L.O. Chua, M. Komuro, T. Matsumoto, The double-scroll family, IEEE Trans Circuits Sy st. 33 (1986) 1072-1118.

[25] C.P. Silva, Shil'nikov's theorem--A tutorial, IEEE Trans Circuits Syst. I 40 (1993) 675-682.

[26] D. Cafagna, G. Grassi, New 3-D-scroll attractors in hyperchaotic Chua's circuit forming a ring, Int. J. Bifur. Chaos 13(10) (2003) 2889--2903.

[27] J. Lu, G. Chen, X. Yu, H. Leung, Design and analysis of multiscroll chaotic attractors from saturated function series, IEEE Trans Circuits Syst. I 51(12) (2004) 2476-2490. 